\title{
IMC based Smith Predictor Design with PI+CI Structure: Control of Delayed MIMO Systems
}

\author{
Ali Dokht Shakibjoo ${ }^{1, a}$, Nastaran Vasegh ${ }^{2}$ and Hassan HosseinNia ${ }^{3}$ \\ 1, 2 Departement of Electrical Engineering, Shahid Rajaee Teacher Training university, Tehran, Iran \\ ${ }^{3}$ Departement of Precision and Microsystems Engineering, Delft University of Technology, The Netherlands
}

\begin{abstract}
In this study a novel structure for time-delay MIMO systems controller design is introduced. In this method decoupled Smith predictor (SP) controller is designed using Internal Model Control structure (IMC). In order to approximate decoupled system, step response model approximation is employed and simulated on MIMO multiple time-delay system. Moreover, to improve system performance from overshoot and rise time perspective, Smith predictor controller is combined with PI+CI structure. Furthermore, to increase system robustness, a low pass filter is designed. Afterwards, the proposed structure is applied to the model of a time-delay MIMO distillation tower system and obtained results are compared to those of a PID controller. Finally, performance of different design methods is evaluated using Integral error criterion (Integral Square Error criterion).
\end{abstract}

\section{Introduction}

Time delay is common in most of industrial processes. It basically results from information, mass and energy transfer phenomena which are known as groups of time delays in simple connected dynamic systems. Probing the impacts of disturbance is time consuming; thus, processes with significant time delay are difficult to be controlled by standard feedback controllers. A considerable amount of recent research works have focused on time delay as it is the most crucial factor affecting control quality. The first instance of time-delay compensator for classic control systems was introduced by Smith in 1957 called Smith predictor. Smith predictor aims to remove time delay from control loop. As a result a time-delay free section is achieved for which an ideal controller can be designed $[1,2]$.

The PI+CI controller consists of two parallel PI and CI controllers [3]. Clegg Integral (CI) is the simplest structure for reset control which increases the phase of system and its stability. Reset action occurs when the input signal is set to zero. Additionally, CI is able to overcome limitations of LTI control system. It was firstly introduced by Clegg in 1985 [4]. To design PI+CI structure PI controller is designed in first step so that the most speed and overshoot could be achieved. Then, nonlinear reset mechanism is added to reduce overshoot. Changing reset coefficient which is between $[0,1]$, the best output, from performance indices perspective, might be derived. To date, most of the processes which have been studied using Smith predictor were single input single output systems. Recently, PI+CI structure with Smith predictor control is designed for time-delay first order system [5]. However, this method is not simulated for MIMO systems. In this paper, Smith predictor controller is designed using internal model control structure. This structure is applied to a distillation tower system. To improve output response $\mathrm{PI}+\mathrm{CI}$ controller is added. Finally, comparing simulation results to PID controller, the potential of this hybrid structure for improving performance indices of MIMO systems is discovered.

After PI $+\mathrm{CI}$ structure and Smith predictor controller are introduced, the Smith predictor controller with Internal Model Control is designed in section 3. Afterwards, adjustment of PI+CI structure is discussed to improve system performance. Then, this structure is applied to a MIMO distillation tower system model. Also, results of desired system are compared to PID controller. At the end, the results are validated using Integral error criterion (Integral Square Error criterion). The final section concludes the paper.

\section{Equivalent structure for Smith predictor controller with Internal Model Control}

In this study an equivalent structure of Internal Model Control together with a Smith predictor compensator are utilized. The structure is depicted in figure 1.

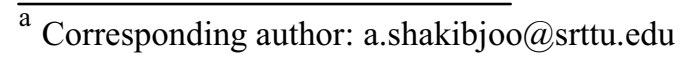




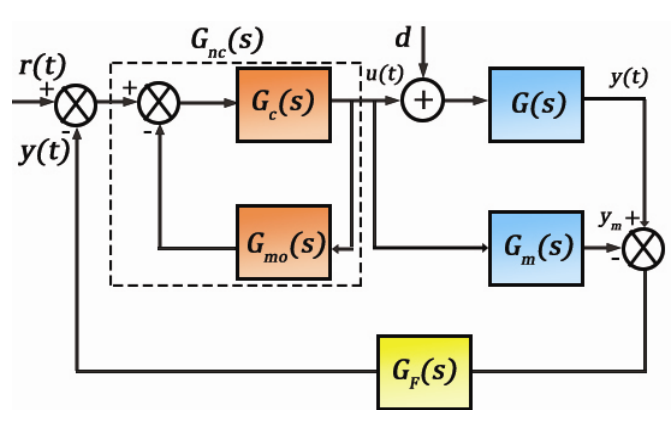

Figure 1. Controller scheme

It is a combination of both Internal Model Control structure (figure 2) and Smith predictor structure (figure 3) and takes advantage of these two structures simultaneously.

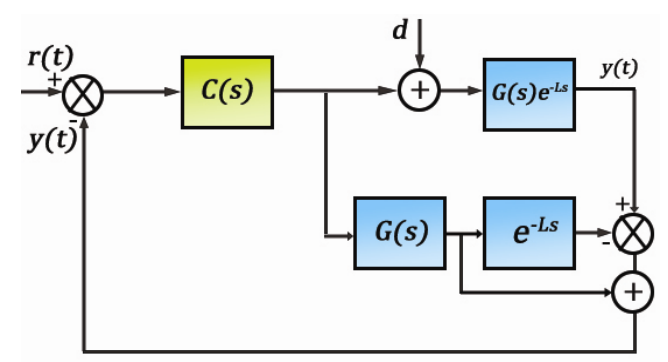

Figure 2. MIC scheme

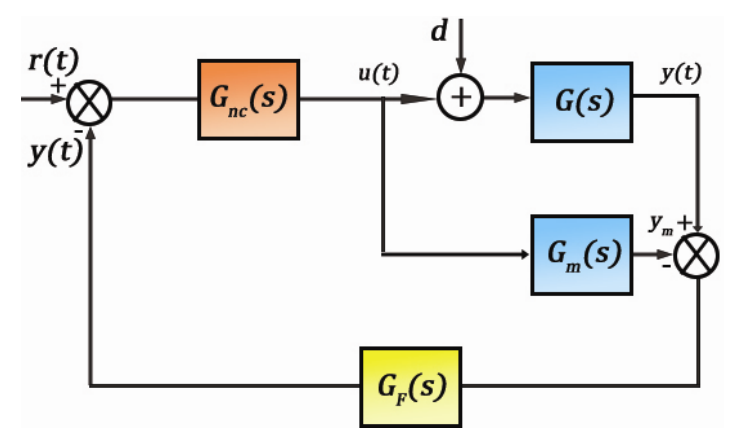

Figure 3. Smith predictor scheme

In the Internal Model Control structure disadvantages such as open loop system, sensitivity to modelling errors and lack of disturbance rejection ability are eliminated. In case of perfect matching between model and process, as well as no disturbance, the system operates as an open loop system which may achieve precise and rapid tracking; whereas, in presence of either mismatch between model and process, or disturbance entrance into the system, it operates as a closed loop system which is able to remove disturbance effects. In our research, the system is a time-delay one; therefore, equivalent structure of Smith predictor together with Internal Model Controller are exploited $[6,7]$.

\section{Designing Smith predictor controller with Internal Model Control}

Consider the undergoing MIMO system transfer function with time delays

$$
G(s)=\left(\begin{array}{cccc}
g_{11}(s) & g_{12}(s) & \ldots & g_{1 m}(s) \\
g_{21}(s) & g_{22}(s) & \ldots & g_{2 m}(s) \\
\vdots & \vdots & \ddots & \ldots \\
g_{m 1}(s) & g_{m 2}(s) & \ldots & g_{m m}(s)
\end{array}\right)
$$

According to the structure demonstrated in figure 1, $G_{n c}(s)$ is Internal Model controller, $G_{c}(s)$ is Smith predictor controller, $G(s)$ is the actual system, $G_{m}(s)$ is system model, $G_{m 0}(s)$ is the system model ignoring time delay and $G_{F}(s)$ is the filter. Initially, using the structure of Internal Model control $G_{n c}(s)$ is designed. Subsequently, Smith predictor controller is derived from Internal Model Controller. Considering figure 1 we have:

$$
G_{n c}(s)=\frac{G_{c}(s)}{1+G_{c}(s) G(s)}
$$

And consequently one may write:

$$
G_{c}(s)=\frac{G_{n c}(s)}{1-G_{n c}(s) G(s)}
$$

To adjust parameters of $G_{c}(s)$ controller, a first order time-delay system is assumed as follows:

$$
\hat{G}(s)=\frac{K e^{-\theta s}}{T s+1}
$$

It could be separated as shown below:

$$
\hat{G}(s)=\hat{G}_{+}(s) \hat{G}_{-}(s), \hat{G}_{+}(s)=e^{-\theta s}, \hat{G}_{-}(s)=\frac{K}{T s+1}
$$

$\hat{G}_{+}(s)$ Includes all time delays and right half plane zeros. Modelling errors must be minimized in internal model controller. Notice that difference between model and process behaviour happens in high frequencies at the end of frequency response. Hence, a series low pass filter is utilized with Internal Model Controller to attenuate mismatch impact. $F(s)$ is the simplest type of low pass filter. This filter is used to assure physical realizability of the internal controller and provides robustness for the system. The simplest filter is as follows

$$
F(s)=\frac{1}{(\lambda s+1)^{n}}
$$

Assuming $\mathrm{n}=1$ and using equations (5) and (6), Internal Model controller is obtained as undergoing equation:

$$
G_{n c}(s)=\hat{G}_{-}(s)^{-1} F(s)=\frac{T s+1}{K(\lambda s+1)}
$$


Utilizing this structure, Smith predictor controller is designed

$$
G_{c}(s)=\frac{T s+1}{K \lambda s} \quad \stackrel{\text { yields }}{\longrightarrow} \quad P I \quad \text { Controller }:=\frac{T}{K \lambda}+\frac{1}{K \lambda s}
$$

The only unknown parameter of the filter is its time constant. It is usually chosen in such a way that its step response is twice faster than open loop response.

Designing Internal Model Controller, transfer function of decoupled process model is derived based on matrix of process model transfer function $G_{m}(s)$ [8].

$$
G_{D}(s)=G_{m}(s)\left[G_{m}(s=0)\right]^{-1}
$$

When the system reaches steady state, $G_{D}(s)$ should be a diagonal matrix to have a perfectly performed static decoupling. That is to say, main diagonal elements of the matrix should be formed of 1 , and non-diagonal elements should be 0 . After decoupling, as the elements of main diagonal include complicated expression with time delays, designing Internal Model controller is difficult. Thus, step response is used to approximate first order timedelay model and main diagonal elements are approximated by first order time-delay model. Then, a controller is designed for each loop using Internal Model Control. As a result, decoupled process controller is obtained as illustrated below:

$$
G_{d c}(s)=\operatorname{diag}\left[G_{d c, 11}(s), \ldots, G_{d c, i i}(s)\right]
$$

Finally, Smith decoupled controller is stated as undergoing equation.

$$
G_{c}(s)=\left[G_{m}(s=0)\right]^{-1} \cdot G_{d c}(s)
$$

The feedback filter is used to address instability problems resulted from model and process mismatch as well as external disturbances. Its transfer function is in the form of equation (11).

$$
G_{F}(s)=\operatorname{diag}\left[\frac{1}{\alpha_{1} s+1}, \ldots \frac{1}{\alpha_{i} s+1}\right]
$$

where $\alpha_{i}(s)$ are adjustment parameters for each loop. They are set to be half of time delay in each loop.

\section{Adjusting $\mathrm{Pl}+\mathrm{Cl}$ Structure}

As shown in figure (4) the PI+CI structure is added to design Smith controller to improve performance indices. To adjust the structure we firstly us MATLAB Simulink to set PI controller for first loop and then CI parameters are derived according to equations (12) where $\tau_{i}, k_{p}$, $\tau_{r}$ and $k_{r}$ are integral time constant, PI gain constant, time constant, and reset control gain, respectively. At last, $\rho$ (reset coefficient) is adjusted to achieve the best response. In the next step, first loop is opened and second

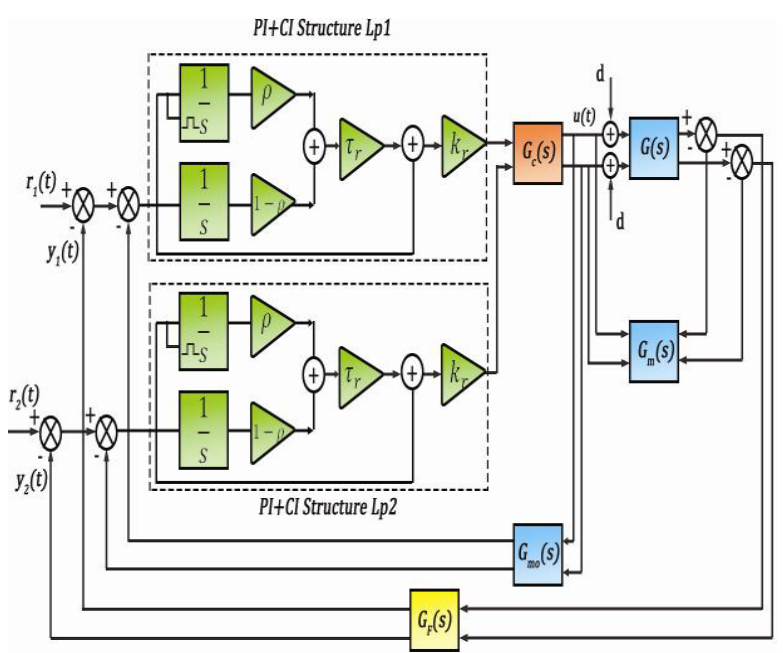

Figure 4. Combined $\mathrm{SP}$ and $\mathrm{PI}+\mathrm{CI}$ reset control

loop controller is designed in a similar manner.

$P I=k_{p}\left(1+\frac{1}{\tau_{i} s}\right), P I D=k_{p}\left(1+\frac{1}{\tau_{i} s}+\tau_{d} s\right), k_{p}=k_{r}, \tau_{r}=\frac{1}{\tau_{i}}$

To give an example, in the next section, time-delay distillation tower system is investigated and Smith predictor controller and $\mathrm{PI}+\mathrm{CI}$ are adjusted for this system.

\section{Equations and mathematics investigating the example}

\subsection{Simulation}

A distillation tower system is utilized. This system is a time-delay MIMO system with a transfer function shown in equation (13) [9].

$$
G_{W B}(s)=\left(\begin{array}{ll}
\frac{12.8}{16.7 s+1} e^{-s} & \frac{-18.9}{21 s+1} e^{-3 s} \\
\frac{6.6}{10.9 s+1} e^{-7 s} & \frac{-19.4}{14.4 s+1} e^{-3 s}
\end{array}\right)
$$

According to mentioned design steps, decoupled matrix is calculated.

$$
G_{m}(s=0)=\left(\begin{array}{cc}
12.8 & -18.9 \\
6.6 & -19.4
\end{array}\right)
$$

Now decoupled matrix is derived using equation 8 : 


$$
\left[G_{m}(s=0)\right]^{-1}=\left(\begin{array}{cc}
0.157 & -0.1529 \\
0.0534 & -0.1036
\end{array}\right)
$$

Then, the first order time-delay model is approximated using step response approximation.

$$
\begin{gathered}
G_{D}(s)=G_{m}(s)\left[G_{m}(s=0)\right]^{-1} \\
=\left(\begin{array}{cc}
\frac{2.0096}{16.7 s+1} e^{-s}-\frac{1.0093}{21 s+1} e^{-3 s} & \frac{-1.9571}{16.7 s+1} e^{-s}+\frac{19580}{21 s+1} e^{-3 s} \\
\frac{1.0362}{10.9 s+1} e^{-7 s}-\frac{1.0360}{14.4 s+1} e^{-3 s} & \frac{-1.0091}{10.9 s+1} e^{-7 s}+\frac{2.0098}{14.4 s+1} e^{-3 s}
\end{array}\right)
\end{gathered}
$$

Then, the first order time-delay model is approximated using step response approximation.

$$
G_{d c 11} \cong \frac{1}{11 s+1} e^{-2 s}, G_{d c 22} \cong \frac{1}{13 s+1} e^{-4 s}
$$

In the following figure decoupled model step response and its approximation can be compared. As can be seen, error between the model and its approximation is negligible. See figure (5).

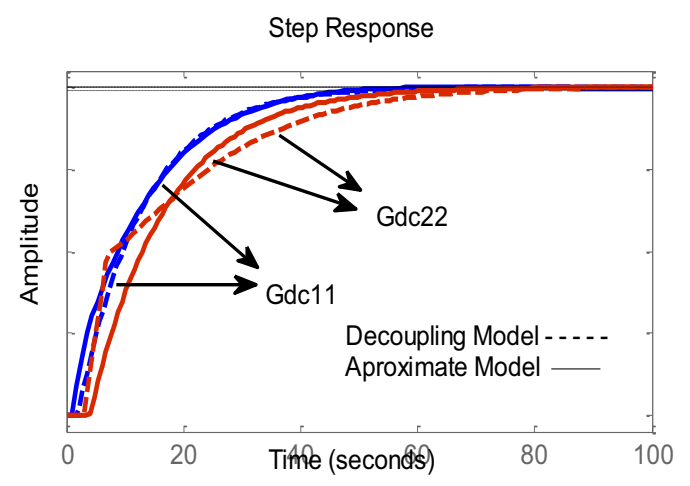

Figure 5. Approximation curve

Decoupled controller is derived as follows based on model approximation

$$
G_{d c}(s)=\left(\begin{array}{cc}
\frac{11 s+1}{\gamma_{1} s} & 0 \\
0 & \frac{13 s+1}{\gamma_{2} s}
\end{array}\right)
$$

$\gamma_{i}$ is chosen so that step response is twice faster than open loop system.

$\gamma_{1}=5.5, \gamma_{2}=6.5$

Decoupled Smith predictor controller is achieved based on equation (11).

$$
G_{c}(s)=\left(\begin{array}{ll}
\frac{0.314 s+0.0285}{s} & \frac{-0.3058 s-0.0235}{s} \\
\frac{0.1068 s+0.0097}{s} & \frac{-0.2072 s-0.0159}{s}
\end{array}\right)
$$

The filter transfer function is calculated as follows according to system time delays.

$$
G_{F}(s)=\operatorname{diag}\left[\frac{1}{1.5 s+1}, \frac{1}{3.5 s+1}\right]
$$

When filter transfer function and Smith predictor controller are designed, PI+CI structure is adjusted.

For ongoing example, PI controller parameters for first loop are calculated as $k_{p}=1.7, \tau_{i}=78.125$; while, the values of these parameters for second loop are equal to 1.15 and 1000, respectively. These parameters are computed using MATLAB Simulink. Substituting in equation (12) CI parameters $\left(k_{r}\right.$ and $\left.\tau_{r}\right)$ are derived for both loops. Then, $\rho$ is set to be 0.9 and 0.8 respectively for first and second loops to improve output response characteristics.

In the next step, the presented structure is replaced with PID controller. The parameters of this controller are adjusted automatically using MATLAB. For the first loop, $k_{p}=0.131, \tau_{i}=14.5$ and $\tau_{d}=-5.11$ and for the second one $k_{p}=-0.088, \tau_{i}=-12.5$ and $\tau_{d}=0.95$.

\subsection{Simulation Results}

The comparison between three controller design methods for output, control and error signals of first and second loops are illustrated in figures (6) and (7). As for tracking, unit step is considered as the input. As it is evident, output response in PID controller has $30 \%$ overshoot and 85 s settling time; however, output response of Smith predictor controller has no overshoot and its settling time is less than PID controller. Furthermore, adding PI+CI structure it achieves faster responses comparing to its rivals. As shown in figure (6) error signal also reaches to its steady state after one oscillation and the proposed structure does not oscillate. As for control signal, although there is larger oscillation in first loop of proposed structure, oscillation of control signal in second loop is smaller.

To investigate the influence of disturbance, step input is applied to the system at $\mathrm{t}=80 \mathrm{~s}$. As demonstrated in figures (6), (7) in case of disturbance, overshoot of PID controller is $10 \%$; nevertheless, in proposed structure overshoot is relatively decreased. It is $6 \%$ for Smith and $2 \%$ for Smith and PI+CI hybrid structure. In conclusion, even though PID controller is the most complete and smartest classic controller with wide-range of applications in industry, our proposed structure is proved to be superior. Adding a simple and cost efficient mechanism, output response of time-delay MIMO system is improved taking into account both step input tracking and disturbance rejection. 

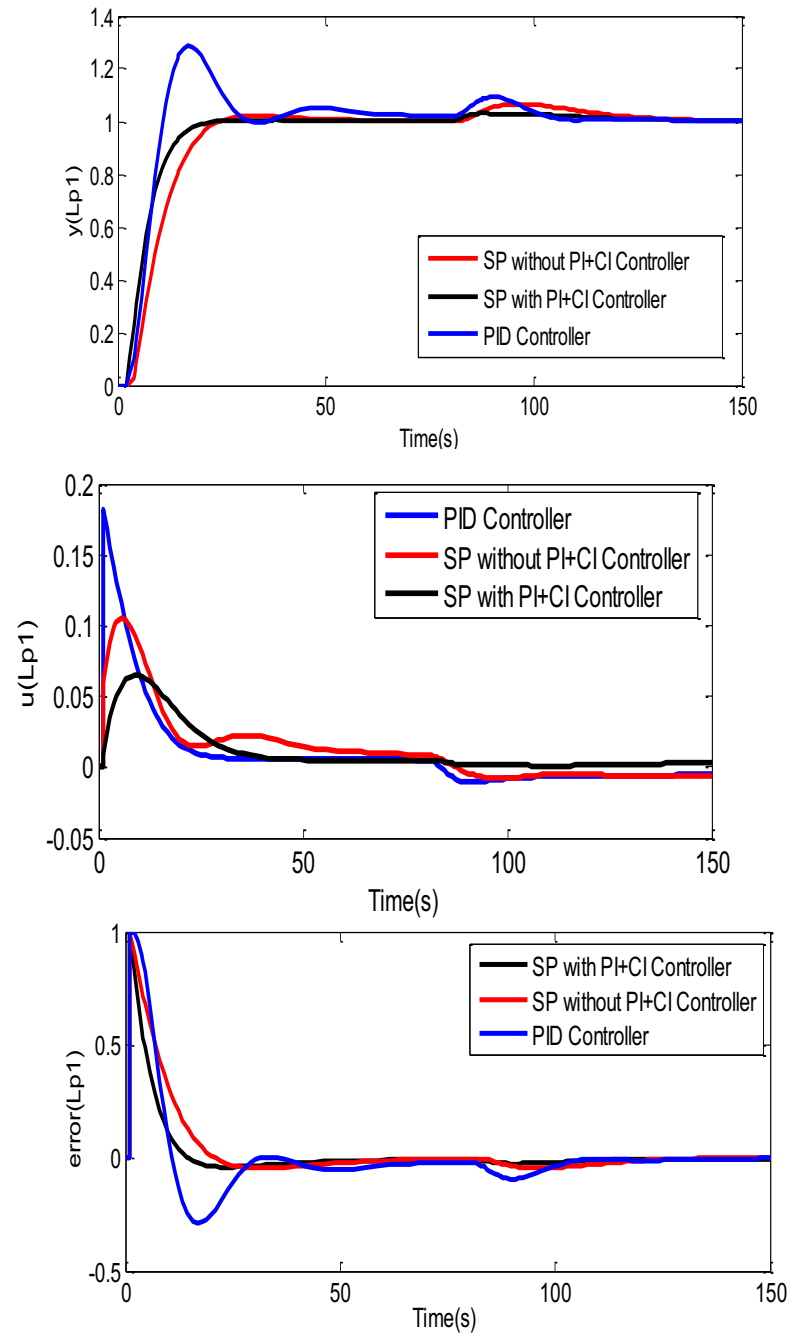

Figure 6. A comparison among SP without $\mathrm{PI}+\mathrm{CI}$ controller with SP with PI+CI controller and PID controller for loop 1.

\section{Validation}

In this section, Integral error criterion (Integral Square Error criterion) is used to validate simulation results.

Table 1 and 2 illustrate performance index for step response tracking and disturbance rejection for three structures (PID, SP, SP-PI+CI). Different design methods are investigated using ISE performance index. Its value is calculated as follows.

$$
I S E_{r_{i}-y_{j}}=\int_{0}^{t}\left(r_{i}-y_{j}\right)^{2} d t, \quad i, j=1,2
$$

Also the sum of ISE values is calculated as shown below: According to table 1 the value of integral square error criterion in tracking $I S E_{r_{1}-y_{1}}$ experiences considerable decrease in proposed structure. Its value was 5.238 with PID controller and it is reduced to 4.127 and 2.397 respectively using SP and SP-PI+CI controllers.
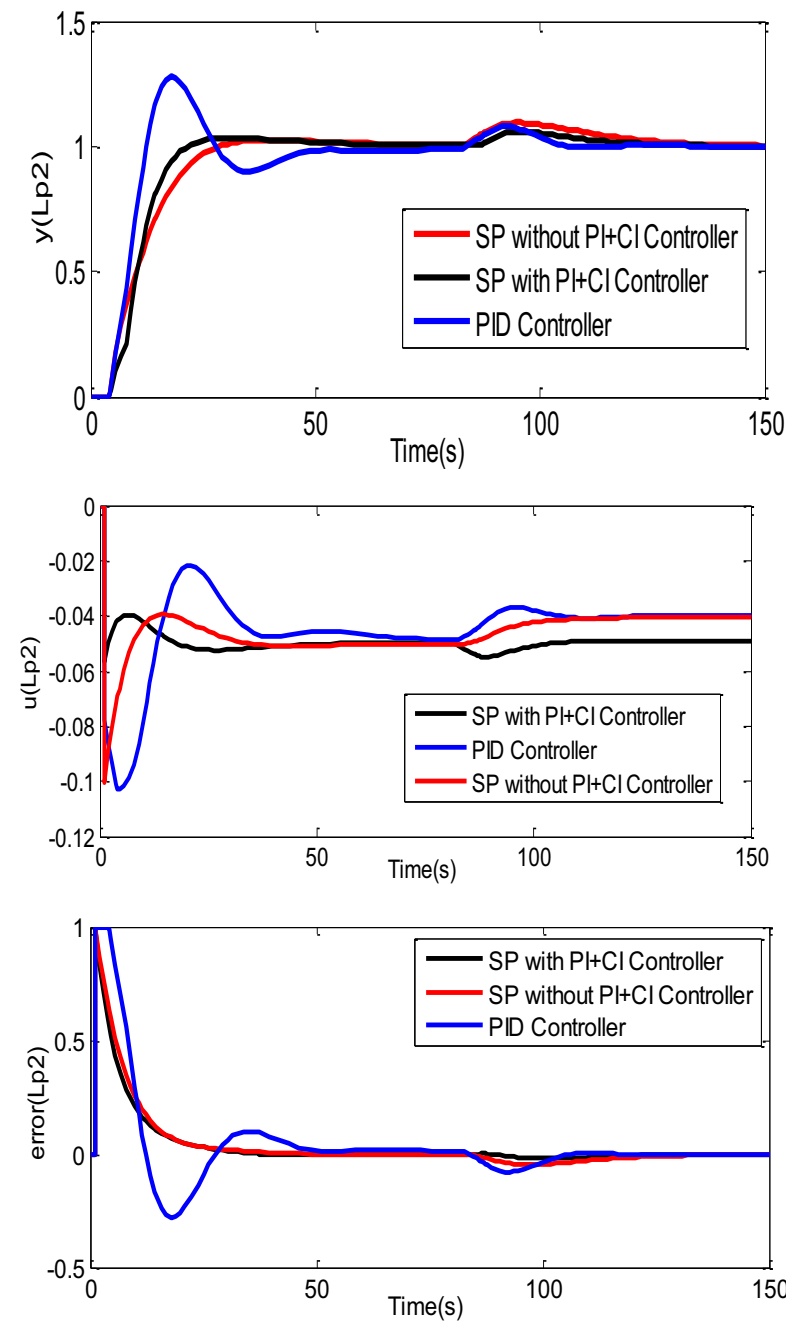

Figure 7. A comparison among SP without $\mathrm{PI}+\mathrm{CI}$ controller with SP with PI+CI controller and PID controller for loop 2

Moreover, $\mathrm{ISE}_{\mathrm{r}_{1}-\mathrm{y}_{2}}$ was 6.525 with PID controller while it changes to 3.35 and 2.774 respectively using SP and SP-PI+CI controllers. In addition, sum of ISE values in proposed structure is much less than the others.

Table 1. A comparison among SP without PI+CI controller, SP with $\mathrm{PI}+\mathrm{CI}$ controller and PID controller in terms of performance indexes for reference tracking

\begin{tabular}{|l|l|l|l|l|l|}
\hline Structure & ISE $_{\mathrm{y} 1-\mathrm{r} 1}$ & ISE $_{\mathrm{y} 2-\mathrm{r} 1}$ & ISE $_{\mathrm{y} 1-\mathrm{r} 2}$ & ISE $_{\mathrm{y} 2-\mathrm{r} 2}$ & $\begin{array}{l}\text { Sum of } \\
\text { ISE } \\
\text { Value }\end{array}$ \\
\hline PID & 5.238 & 6.595 & 5.238 & 6.595 & 23.66 \\
\hline SP & 4.127 & 3.355 & 4.127 & 3.355 & 14.964 \\
\hline $\begin{array}{l}\text { SP- } \\
\text { PI+CI }\end{array}$ & 2.397 & 2.774 & 2.397 & 2.774 & 10.342 \\
\hline
\end{tabular}

The Integral square error criterion is shown in table 2 in presence of disturbance. A significant reduction is observed in ISE in all four conditions of proposed structure comparing to PID controller. The sum of ISE 
was 23.945 in PID method and it changes to 15.149 and 10.395 respectively with Smith and proposed structure.

Table 2. A comparison among SP without PI+CI controller with SP with PI+CI controller and PID controller in terms of performance

\begin{tabular}{|l|l|l|l|l|l|}
\hline \multirow{2}{*}{ Structure } & ISE $_{\mathrm{y} 1-\mathrm{r} 1}$ & ISE $_{\mathrm{y} 2-\mathrm{r} 1}$ & ISE $_{\mathrm{y} 1-\mathrm{r} 2}$ & ISE $_{\mathrm{y} 2-\mathrm{r} 2}$ & $\begin{array}{l}\text { Sum of } \\
\text { ISE } \\
\text { Value }\end{array}$ \\
\hline PID & 5.327 & 6.65 & 5.327 & 6.65 & 23.945 \\
\hline SP & 4.182 & 3.444 & 4.138 & 3.385 & 15.149 \\
\hline $\begin{array}{l}\text { SP- } \\
\text { PI+CI }\end{array}$ & 2.405 & 2.813 & 2.401 & 2.776 & 10.395 \\
\hline
\end{tabular}

\section{Conclusions}

In this study, a novel structure for MIMO system controller design is proposed. In this structure decoupled Smith predictor controller is employed combined with Internal Model Control structure. Then, output characteristics were improved by adjusting parallel PI+CI structure. Internal Model Control structures suffer from steady state errors in case of disturbance; whereas, our proposed method overcomes this problem. To increase robustness, low pass filter was designed. The proposed structure was compared to PID controller while applied to a distillation tower system. This comparison revealed that our proposed method is advantageous due to smaller overshoot and faster tracking. Additionally, based on simulation results and considering disturbance rejection, our novel structure had better dynamic performance and stability in comparison with other structures. In case of mismatch between system model and the process our proposed method showed better robustness. At last, Integral Square Error criterion is used to validate system performance.

\section{References}

1. J. Normey-Ricoa, E. Camacho, Control Eng. Pract, 16, 407-428 (2008).

2. J. Normey-Ricoa, E. Camacho, Control of dead-time processes (Berlin Springer 2007).

3. A. Baños, A. Barreiro, Reset control systems, (Advances Industrial Control, Springer 2012).

4. J. C. Clegg, Trans. AIEE, 77, 41-42 (1958).

5. J. Moreno, J. L. Guzman, J. E. Normey-Rico, A. Banos, M. Berenguel,IFAC PID 12 , Brescia (Italy), March 28-30 (2012).

6. D.E. Rivera, M. Morari, S. Sigurd, ind. Chem. Process Des, 25, 252-265 (1986).

7. W.Tan, T. Chen, J. Pocess Control, 13, 203-213 (2013).

8. G. Juan, F. Morilla, J. Process Control, 13, 978-983 (2011).

9. R. K. Wood, M. W. Berry, Chem. Eng. Sci, 28, 1707-1717 (1973). 